\title{
CIERRE DE PERFORACIÓN A NIVEL DEL TERCIO APICAL CON BIODENTINE: REPORTE DE UN CASO
}

\section{INTRODUCCIÓN}

Las perforaciones radiculares son percances que se pueden presentar a lo largo de la terapia pulpar y pueden comprometer el pronóstico del órgano dental. $(1,2)$ En estos incidentes se presentan numerosos factores que deben ser tomados en cuenta para la resolución de los mismos, como son el tamaño, ubicación y tiempo que se presente dicha perforación (3).

Biodentine fue introducido por la casa Septodont en el 2009 compuesto por silicato tricálcico, silicato dicálcico, carbonato de calcio, óxido de relleno, óxido de hierro, óxido de zirconio, cloruro de calcio y polímero hidrosoluble, ha demostrado ser uno de los más eficientes debido a su biocompatibilidad y mayor capacidad de sellado como un sustituto de la dentina.

\section{RESUMEN}

Antecedentes: El presente caso clínico se desarrolló en la clínica de posgrado de la Facultad de Odontología de la Universidad Central del Ecuador, en donde se realizó el sellado de la perforación a nivel del tercio apical, el procedimiento se ejecutó con Biodentine (Septodont) el cual es un nuevo cemento de silicato de calcio que presenta propiedades biocompatibles y bioactivas que permiten la remineralización del defecto óseo (4).

Objetivo: Presentar el reporte de un caso acer- ca de una perforación a nivel apical tratada con Biodentine formando así un tope apical.

Conclusiones: El Biodentine es un cemento biocerámico el cual ha mostrado excelentes resultados tanto en su biocompatibilidad, microdureza, tiempo de trabajo, entre otras características, por lo que resultó favorable y de gran éxito en el tratamiento y evolución del caso que se presenta; siendo así este material una elección primordial para el especialista. (5). 


\section{Clínica}

\section{Primera Cita}

Paciente de sexo femenino, 19 años de edad acude a la clínica de Posgrado de Facultad de Odontología cuyo motivo de consulta fue la revisión de la pieza 1.1 previamente iniciado el tratamiento. La paciente refiere que días anteriores, al presentar su cara hinchada, se acercó al centro de salud donde le realizaron la apertura de la cavidad para que drene y recetaron amoxicilina $500 \mathrm{mg}$ c/8h por 7 días, hasta que acuda a una consulta de especialidad de endodoncia, a donde a fue remitida.

Una vez realizada la historia clínica y la anamnesis se procede a tomar radiografía preoperatoria (Fig.1) en donde se observó, a nivel del tercio apical dirigido hacia mesial, una sombra radiolúcida compatible con una perforación de aproximadamente dos $\mathrm{mm}$ de ancho; se explica a la paciente el percance encontrado y una vez obtenido el consentimiento informado se inicia la terapéutica.

Se realiza aislamiento absoluto, posteriormente se perfeccionó la cavidad de acceso y se neu- tralizó irrigando con $\mathrm{NaOCl}$ al 5,25\%, se efectúa la toma de longitud aparente en la radiografía mediante el método de Ingle, siendo esta inicialmente de $18 \mathrm{~mm}$, y se comprobó dicha longitud mediante la utilización del localizador apical (J. Morita) y una lima k\#15, pero se presentó un pequeño sangrado por lo que se le derivó para tomar una nueva radiografía para comprobar la localización de la lima en el conducto, y se pudo observar que dicho instrumento emergía por la excavación provocada en el tratamiento previo (Fig.2).

Al observar que la lima tomó el camino de la perforación se empezó a recanalizar el conducto, desde una lima \#6 precurvada hacia distal hasta la lima \# 15, volviendo a tomar la longitud de trabajo correcta siendo esta de $20,5 \mathrm{~mm}$, irrigando siempre entre cada lima con $\mathrm{NaOCl} 5,25 \%$. Se saturó el conducto con medicación intracanal a base de una pasta de $\mathrm{Ca}(\mathrm{OH}) 2$ (Ultracal) (Fig.3) y se le citó en 8 días.

\section{Segunda Cita}

Se verificó que no existía sintomatología y se procedió a retirar el hidróxido de calcio, finalizando la preparación químico mecánica con lima $\mathrm{k}$ \# 50 hasta la longitud de trabajo de $20,5 \mathrm{~mm}$ acompañado de $\mathrm{NaOCl} 5,25$ entre cada lima,

culminando con limas Protaper manual para así dar conicidad al conducto. Hidróxido de calcio (Ultracal) se colocó intracanal ocho días previos a la culminación del procedimiento. 


\section{Tercera Cita}

Se recupera la longitud de trabajo y se observa radiográficamente que no hayan quedado restos de hidróxido de calcio dentro del canal, a continuación se realizó la irrigación final más activación con $\mathrm{Na}$.OCI 5,25\%, edta $17 \%$ por 3-5 minutos, $\mathrm{Na} . \mathrm{OCl} 5,25 \%$ para posteriormente continuar con la prepararación de Biodentine (Septodont) de acuerdo a la indicación de la casa comercial, el mismo que se llevó al conducto con ayuda de un porta amalgama y con los Plugger Machtou (Dentsply Maillefer), se compactó a nivel apical, esperamos el fraguado del material y finalmente se tomó una radiogra- fía para comprobar el selle de la perforación apical y procediendo con la obturación final.

La obturación final se realizó con cono maestro $\mathrm{N \#} 50$ y conos accesorios MF y FF de la casa Becht y Meta respectivamente mediante la técnica de condensación lateral, se efectuó la toma radiográfica previo el corte del penacho (Fig. 4) y finalmente se selló la entrada del conducto con ionómero de vidrio de fotocurado (GC Fuji II LC), a continuación se generó la radiografía final postoperatoria (Fig. 5) y se remitió a la paciente para la rehabilitación del órgano dentario.

Cuatro meses después se realizan controles

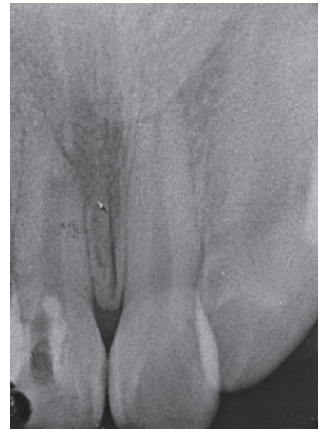

FIG 1

Radiografía Preoperatoria

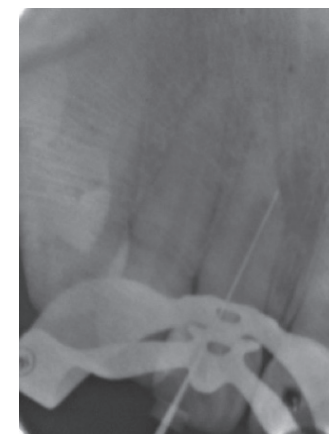

FIG 2: Conductometría

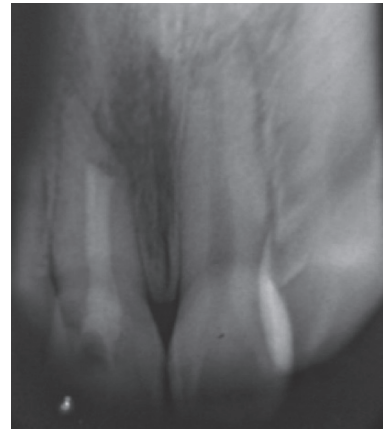

FIG 3:

Medicación intracanal

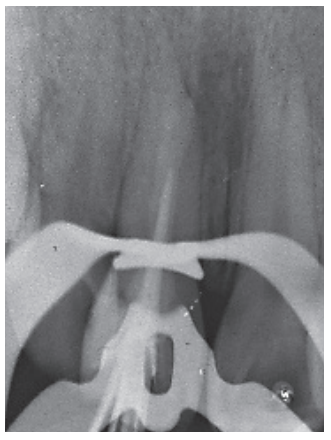

FIG 4:

Penacho

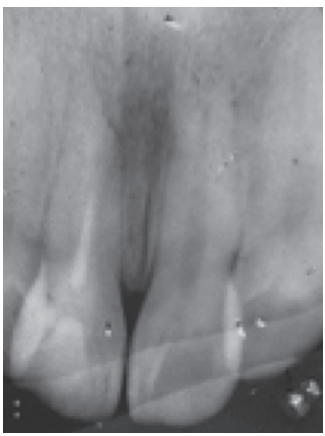

FIG 5: Radiografía Final
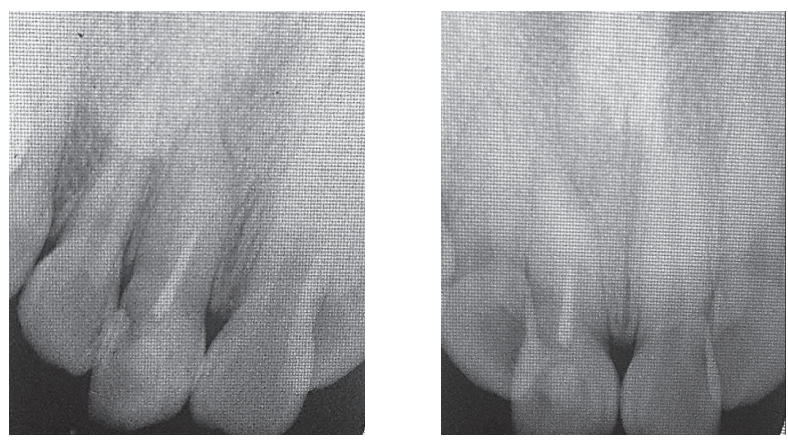

FIG 6:

Control 4 meses después

\section{Conclusiones}

El pronóstico de las perforaciones dependerá de distintos factores como: la infección bacteriana en el sitio de la lesión, el tiempo transcurrido desde el percance hasta el tratamiento, la ubicación y el tamaño.

Biodentine demostró ser un excelente material biocompatible y que ayudó a la evolución de la lesión, con su capacidad de interface con la dentina y su bioactividad se obtuvo óptimos resultados.

Biodentine es un material nuevo que ha demostrado ser un buen sustituto de la dentina.

Por su bajo costo y alta efectividad se ha convertido en el material de elección para distintos tratamientos a nivel endodóntico y a nivel Odontológico en general. 


\section{Discusión}

Las perforaciones radiculares son percances que se pueden presentar durante el tratamiento endodóntico, pudiendo inferir en el pronóstico de la pieza a tratarse $(6,7,8,9)$ teniendo como consecuencia una posible afección a los tejidos periapicales (3). Sin embargo en este estudio hemos podido observar que dicho percance se puede solucionar si lo tratamos de la manera adecuada, obteniendo así éxito con un paciente asintomático y sin lesión periapical aparente de acuerdo a esta base sustentada, en este caso se decidió realizar el selle de la perforación con Biodentine.

Biodentine es un nuevo material que se ha presentado como el mejor sustituto de la dentina, pudiéndolo utilizar en distintas situaciones a nivel endodóntico, Septodont (2012), a pesar de que varios autores indican que MTA es uno de los mejores cementos de uso en endodoncia por su radiopacidad, composición, biocompatibilidad y su bioactividad $(10,11)$, hay otros autores que discrepan con esto, mencionando desventajas importantes a nivel de tejidos dentarios como son su pigmentación, el difícil manejo y largo tiempo de fraguado $(12,13)$.

En este caso pudimos observar en el plazo de cuatro meses aproximadamente una evolución favorable, con una reducción significativa de la radiolucencia, que en un principio se encontró a nivel periapical y con una sintomatología totalmente ausente, concordando así con distintos autores quienes indican que con una técnica adecuada de instrumentación (14), medicación intracanal para eliminar bacterias y un buen protocolo de irrigación (12) se consigue el éxito. Biodentine es un excelente cemento sellador por su adhesión a la dentina, sus indudables propiedades biominerales y su biocompatibilidad con los tejidos periapicales (10).

\section{BIBLIOGRAFÍA}

1. Fuss Z, Trope M. Root perforations: classification and treatment choices based on prognostic factors. Endod Dent Traumatol 1996; 12: 255-264.

Adel M, Tofangchiha M, Yeganeh LA, Javadi A, Khojasteh AA, Majd NM. Diagnostic accuracy of cone-beam computed tomography and conventional periapical radiography in detecting strip root perforations. J Int Oral Health 2016;8(1):75-79.

3. Tsesis I, Rosenberg E, Faivishevsky V, et al. Prevalence and associated periodontal status of teeth with root perforation: a retrospective study of 2,002 patients' medical records. J Endod 2010;36:797-800.

Graziela Garrido Mori., et al. Biocompatibility Evaluation of Biodentine in Subcutaneous Tissue of Rats. Journal of endodontics. 2014. 40 (9).

5. Guneser MB, Akbulut MB, Eldeniz AU. Effect of various endodontic irrigants on the push-out bond strength of Biodentine and conventional root perforation repair materials. J Endod 2013;39:380-4.

6. Tsesis I, Fuss Z. Diagnosis and treatment of accidental root perforations. Endod Topics 2006;13:95-107. 7. Ree M, Schwartz R. Management of perforations: four cases from two private practices with medium- to long-term recalls. J Endod 2012;38:1422-7.

8. Marion JJC, Duque TM, Santos TS, Manhaes FC. The use of MTA in the treatment of cervical root perforation: case report. Dental Press Endod. 2013 MayAug;3(2):96-101.
9. Ramos JC., et al. 1 -year In Vitro Evaluation of Tooth Discoloration Induced by 2 Calcium Silicate-based Cements. .Journal of endodontics. 2016 Sep;42(9) 10. Haghgoo R., et al. An In vitro Comparison of Furcal Perforation Repaired with Pro-root MTA and New Endodontic Cement in Primary Molar Teeth- A Microleakage Study. J Dent Shiraz Univ Med Sci., March 2014; 15(1): 28-32

11. Bogaerts P. Treatment of root perforations with calcium hydroxide and SuperEBA cement: a clinical report. Int Endod J 1997; 30: 210-219.

12. Kailing Siew., et al.Treatment Outcome of Repaired Root Perforation: A Systematic Review and Meta-analysis. Journal of endodontics. 2015; 41 (11). 1795-1804.

13. Machado R, Back EDEE, Reis G, Stiz R, Tomazinho LF, Simi Júnior J, Vansan LP. latrogenic apical root perforation performed during retreatment of a mandibular central incisor: A five-year follow-up case study. Dental Press Endod. 2014 Sept-Dec;4(3):53-6.

14. L. Han, T. Okiji.et al. Uptake of calcium and silicon released from calcium silicate-based endodontic materials into root canal dentine. International endodontic Journal. 2011. 44 (12). 1081-1087 